\title{
EELS Analysis Of Lithiation/Delithiation Reactions In $\mathrm{LiFePO}_{4}$
}

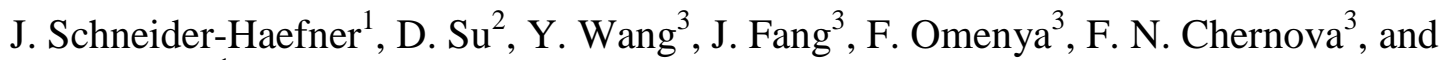 \\ F. Cosandey ${ }^{1}$ \\ ${ }^{1}$ Department of Materials Science \& Engineering, Rutgers University, Piscataway, NJ 08854 \\ ${ }^{2}$ Center for Functional Nanomaterials, Brookhaven National Laboratory, Upton, NY 11973 \\ ${ }^{3}$ Department of Chemistry, Binghamton University, Binghamton, New York 13902
}

$\mathrm{LiFePO}_{4}$ has emerged as an important cathode material for Li-ion batteries because of it stability and high rate capabilities. It is now well established that lithiation-delithiation occurs via a twophase reaction. At high charge/discharge rates, the process of nucleation and growth of a two phase reaction is too slow and a non-equilibrium single phase reaction has been proposed followed by relaxation into $\mathrm{LiFePO}_{4}$ and $\mathrm{FePO}_{4}$ end product phases [1]. In this study, we studied reaction mechanisms and determined the spatial distribution of lithiated/delithiated phases by STEM/EELS spectrum imaging.

$\mathrm{LiFePO}_{4}$ particles from partially charged or discharge electrodes were observed with a cold cathode field emission Hitachi HD2700C STEM and Gatan Enfina EELS spectrometer. The energy resolution of the combined STEM/EELS system was $0.5 \mathrm{eV}$. The energy was calibrated with respect to the main O-K peak at $539 \mathrm{eV}$. Typical EELS spectrum for $\mathrm{LiFePO}_{4}$ and $\mathrm{FePO}_{4}$ are shown in Fig. 1a and $1 \mathrm{~b}$ respectively. A characteristic feature of delithiated $\mathrm{FePO}_{4}$ phase is the presence of an oxygen pre-peak marked by an arrow in Fig.1b. The existence of this $\mathrm{O}$ prepeak has been attributed to a transition from $\mathrm{O} 1 \mathrm{~s}$ to $2 \mathrm{p}$ hybridized state with $\mathrm{Fe} 3 \mathrm{~d}$ [2]. In addition the change in $\mathrm{Fe}$ valence state from $\mathrm{LiFe}^{2+} \mathrm{PO}_{4}$ to $\mathrm{Fe}^{3+} \mathrm{PO}_{4}$ is accompanied with a shift to higher energy of $\mathrm{Fe}-\mathrm{L}_{3}$ peak position of about $1.5 \mathrm{eV}$. In this study we have quantified the existence of these two lithiated and delithiated phases from the shift in $\mathrm{Fe}-\mathrm{L}_{3}$ peak energy, $\mathrm{Fe} \mathrm{L}_{3} / \mathrm{L}_{2}$ peak intensity ratio and from quantification of normalized pre-O peak intensity. Measurements made from about 50 particles reveal two clusters of data with average $\mathrm{Fe}-\mathrm{L}_{3}$ peak energy of $708.2 \mathrm{eV}$ and $709.8 \mathrm{eV}$ with $\mathrm{O}$ pre-peak intensity ratio of 0.037 and 0.16 respectively. These two data clusters correspond to the lithiated $\mathrm{LiFePO}_{4}$ and delithiated $\mathrm{FePO}_{4}$ phases. The spectrum images of the lithiated $\mathrm{LiFePO}_{4}$ and delithiated $\mathrm{FePO}_{4}$ expressed as the normalized $\mathrm{O}$ pre-peak intensity are shown in Fig. $2 \mathrm{a}$ and $2 \mathrm{~b}$ respectively revealing uniform lithiation throughout the particles, i.e. the particles are either fully lithiated or fully delithiated in accordance with the non-equilibrium solid solution transformation path followed by relaxation. An ADF-STEM image taken from an area with many particles and the corresponding phase distribution map are shown in Fig.3a and $3 \mathrm{~b}$ respectively, revealing a non-uniform distribution of phases with agglomeration of fully lithiated and delithiated regions that include many particles.

\section{References}

[1] F. Omenya et al. Adv. Energy Mater. 4 (2014) 1401204 (9pp)

[2] M.K. Kinyanjui et al. J. Phys. Condens.Matter, 22 (2010) 275501 (8pp)

[3] Supported by NECCES a DOE-BES-EFRC funded center under Grant DE-SC0001294. 

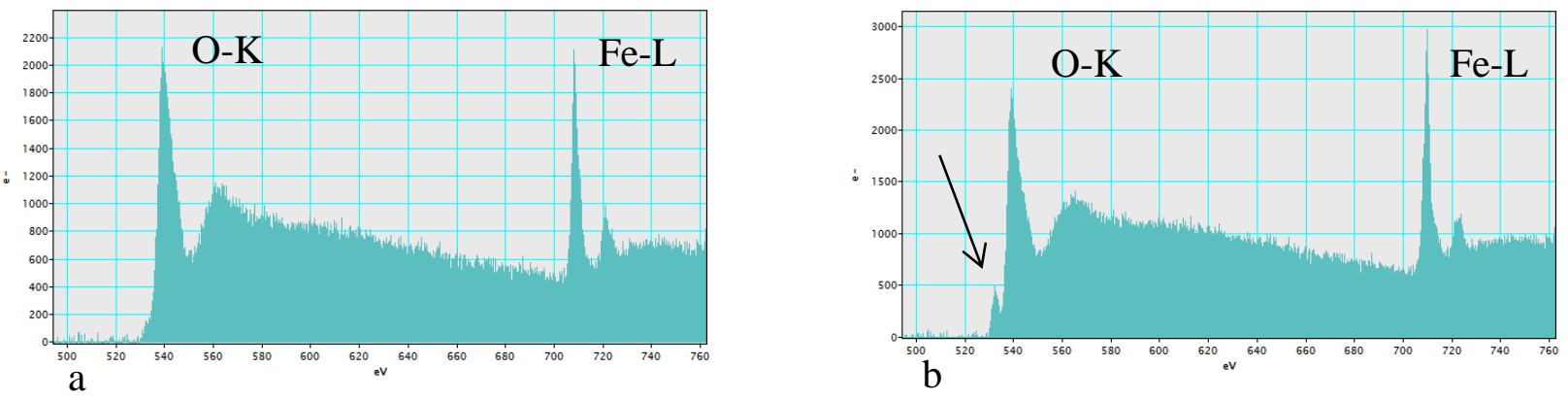

Fig. 1. EELS spectra of (a) fully lithiated LiFePO4 and (b) fully delithiated $\mathrm{FePO}_{4}$ showing characteristic O-K prepeak marked by an arrow.
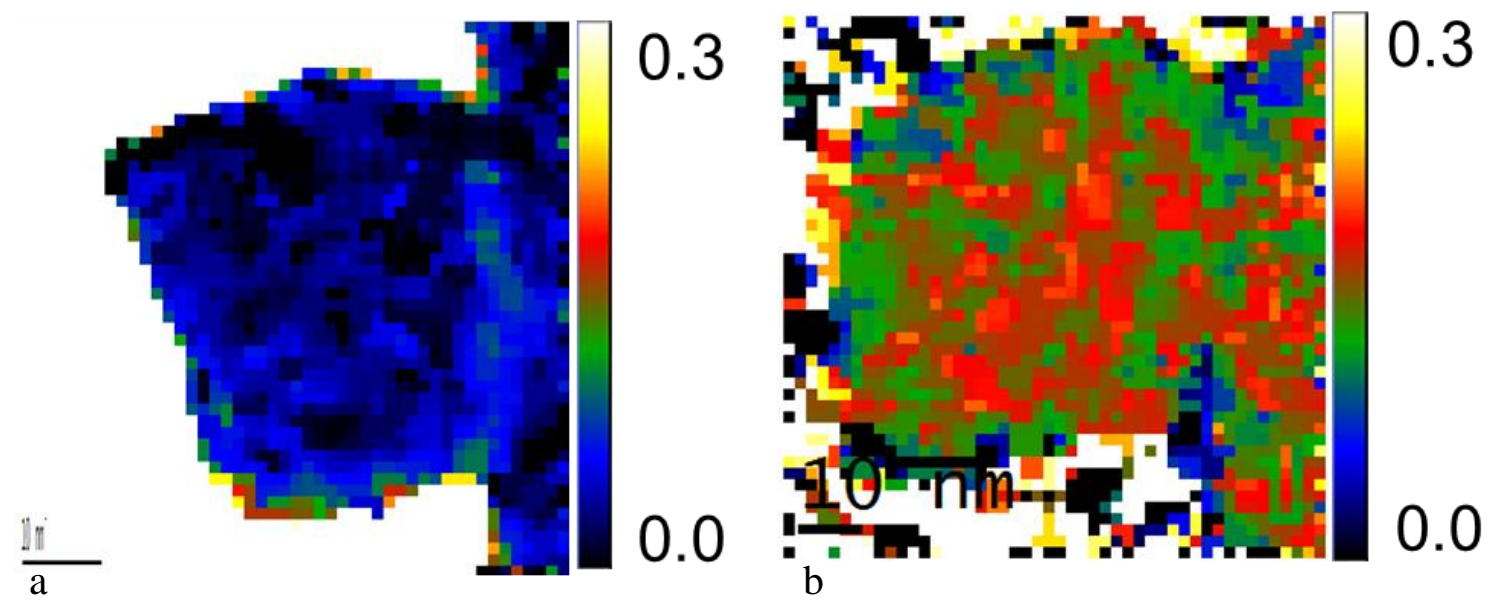

Fig.2. Normalized oxygen pre peak intensity map for (a) $\mathrm{LiFePO}_{4}$ and (b) $\mathrm{FePO}_{4}$

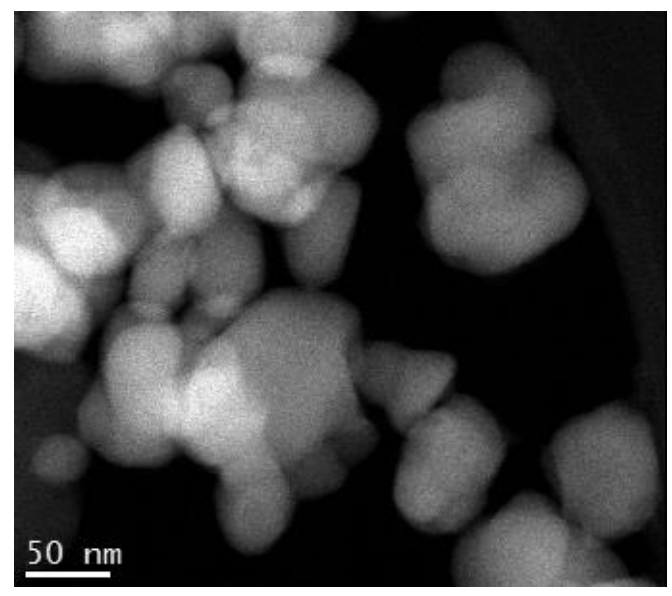

a

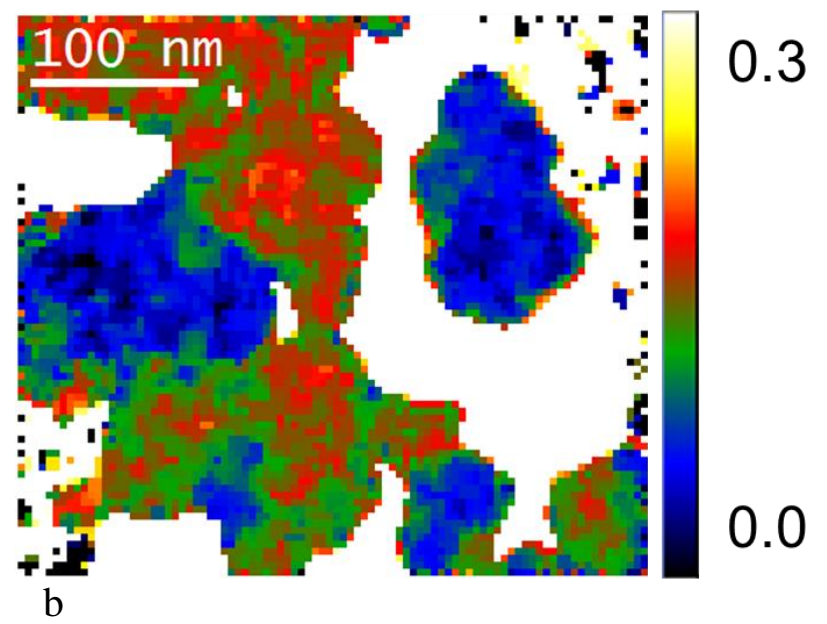

Fig.3. (a) ADF-STEM image of 50\% delithiated $\mathrm{LiFePO}_{4}$ and (b) corresponding normalized oxygen pre peak intensity map. 